\title{
AÇÃO INDESEJÁVEL DE TERAPÊTICA ANTIEPILÉPTICA EM PACIENTE COM EPILEPSIA E DESCARGAS FOCAIS DE "MATURAÇÃO”
}

\author{
José Geraldo Speciali * \\ Michel PierRe Lison **
}

A participação da terapêutica anticomicial na indução de epilepsias graves nâo foi definida, motivo pelo qual julgamos de interesse relatar a observação seguinte.

O BSER V A ÇÃ O

S.M.R.O., de 4 anos, sexo feminino, branca, foi atendida pela primeira vez no Serviço de Neurologia em 31 de maio de 1971. Com a idade de 1 ano e 7 meses apresentara "nefrite" e, no $60^{\circ}$ dia da moléstia, hipotonia com cianose por cerca de 1 hora. Passados dois dias, surgiram, ao despertar, crises tônicas generalizadas diárias com taquipnéia e palidez seguidas por período de uma a duas horas de medo intenso, principalmente de ficar só e em lugares altos. Apareceram auto e alo-agressividade, diminuição no tempo de sono e terror noturno. Em janeiro de 1970 houve melhora das crises após a introdução de primidona e fenobarbital. A partir de maio de 1971 foram administrados $10 \mathrm{mg}$ de nitrazepam e 50 de fenobarbital por dia, aumentando a freqüência das crises e melhorando o comportamento. Antecedentes - Nasceu de parto normal, com discreta cianose; desenvolvimento psicomotor normal até a ocorrência da "nefrite" deixando de andar e falar após a crise de hipotonia. Recuperou a deambulação aos dois anos de idade. Exame clinico-neurológico - Criança com retardo psicomotor, deambula, conhece familiares, emite sons ininteligíveis, não reconhece brinquedos nem se alimenta por si, não obedece ordens simples, não controla esfincteres. Exames complementares - No sangue: sódio 140,6 mEq/1; potássio 4,0 mEq/1; glicose $72,6 \mathrm{mg} / 100 \mathrm{ml}$; colesterol $204 \mathrm{mg} / 100 \mathrm{ml}$; uréia $32,4 \mathrm{mg} / 100 \mathrm{ml}$; creatinina $0,6 \mathrm{mg} / 100 \mathrm{ml}$. Hemograma: hemácias $4.000 .000 \mathrm{~mm}^{3}$; hemoglobina $11,1 \mathrm{~g} / 100 \mathrm{ml}$; leucócitos $4.000 / \mathrm{mm}^{3}$ (neutrófilos segmentados $42,0 \%$, eosinófilos $22,0 \%$, linfócitos $30 \%$, monócitos $6,0 \%$ ). Nas fezes: ovos de Ascaris lumbricóicles. Na urina: ausência de substâncias redutoras e traços (indosáveis) de proteínas; 8 a 10 leucócitos e 1 a 2 hemácias por campo. No líquido céfalorraqueano (punção lombar): células $0,6 \mathrm{~mm}^{3}$; proteínas $23 \mathrm{mg}$ / $100 \mathrm{ml}$; cloretos $720 \mathrm{mg} / 100 \mathrm{ml}$; reações de Wassermann e para cisticercose negativas. Radiografias do crânio normais. Exame eletrencefalográfico (EEG): pontas, isoladas ou agrupadas, de projeção assincrona em áreas occipital esquerda e frontorolândico-parietais com polaridade positiva nas projeçōes anteriores e negativa nas rolandico-parieto-occipitais (Fig. 1).

Evolução - Em outubro de 1971 foram acrescentados $100 \mathrm{mg}$ de difenilhidantoina. Houve redução do número das crises estimada em 40\%. O EEG, em 17 de novembro, revelou ponta-ondas irregulares, de $3 \mathrm{c} / \mathrm{seg}$ na região rolândico-parietal es-

Departamento de Neuropsiquiatria e Psicologia Médica da Faculdade de Medicina de Ribeirão Preto da Universidade de São Paulo (Prof. J. Armbrust-Figueiredo): * Auxiliar de Ensino; * Professor Adjunto. 


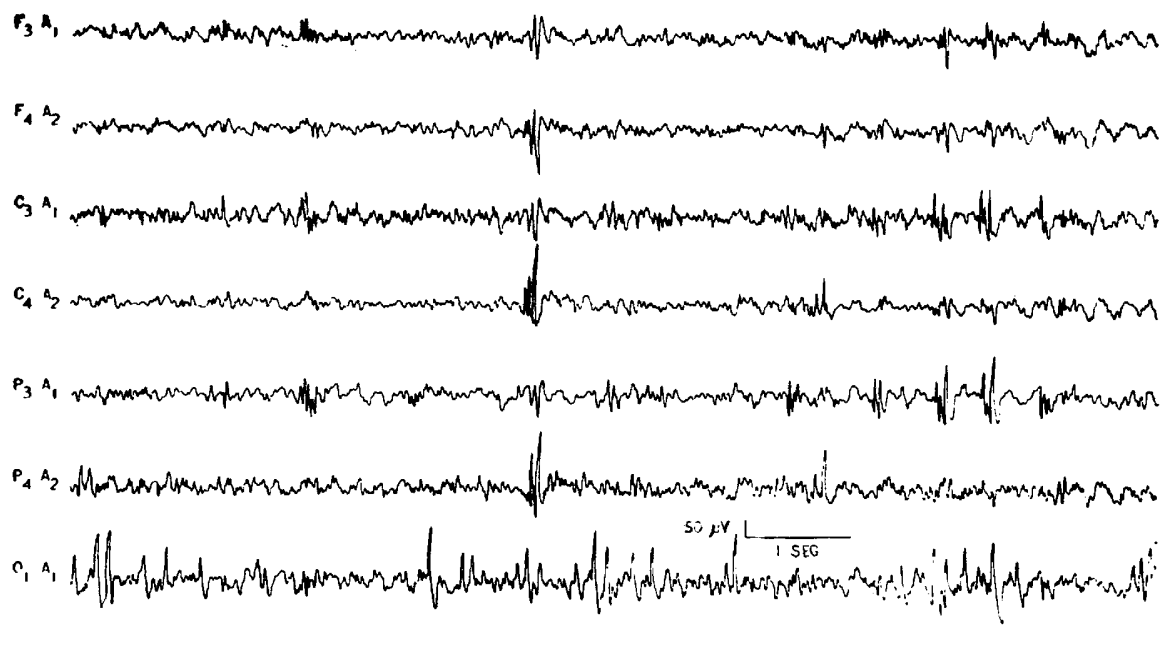

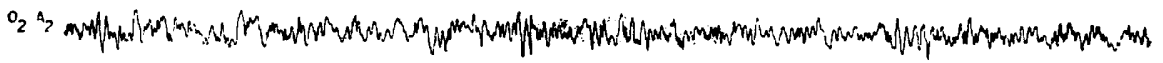

Fig. 1 - Caso S.R.M.O. - Sono barbitúrico. Antes do tratamento com difenilhidantoina. Pontas freqüentes, de projesc̃o assíncrona em áreas rolandico-parientais de ambos os hemisféricos cerebrais e na região occipital esquerda.

querda e pontas rolândicas isoladas à direita. Em dezembro substituiu-se o nitrazepam por clonazepam, três miligramas por dia, havendo controle das crises por dois meses e piora do comportamento. Em fevereiro e marco de 1972, com o aumento da dose de difenilhidantoina para $200 \mathrm{mg}$ por dia, não ocorreram manifestacões epilépticas mas nos dois meses seguintes foram observadas 6 crises tônicas. Em julho apresentava ataxia e hipertrofia gengival. Ocorreram duas a três crises atônicas à direita por dia com queda ocasional. O EEG, em 14 de agosto, revelou atividade de fundo lenta, ponta-ondas, ponta-ondas agudas e ponta-ondas lentas irregulares, pseudorritmicas e difusas predominando no hemisfério cerebral esquerdo (Fig. 2). Suspensa a difenilhidantoina, as crises atônicas desapareceram em uma semana. O EEG, em 28 de agosto, evidenciou pontas e ponta-ondas irregulares e difusas predominando na região rolândico-parietal esquerda (Fig. 3). Em 18 de setembro foram registradas pontas, isoladas ou por surtos, nas regiões fronto-rolândico-parietais e occipital esquerda. A polaridade das pontas frontais voltou a ser invertida em relacão à das rolândico-parietais (Fig. 4). A recidiva das crises tônicas em fins de setembro obrigou a reintrodução da difenilhidantoina, $100 \mathrm{mg}$ por dia. Com essa medida a criança apresentou apenas 4 crises num periodo de 2 meses.

\section{COMENTARIOS}

As características clínicas das epilepsias, sua freqüência e seu prognóstico dependem não somente da gravidade da lesão ou do distúrbio funcional epileptógeno primário mas também do estadio de maturação do encéfalo, da incidência de novas agressões encefálicas, diretas ou indiretas, de fatores extrínsecos (ciclos nictemérico e hormonal) e intrínsecos (genéticos, vasculares ou metabólicos, repetição de crises em estados de mal epilépticos 


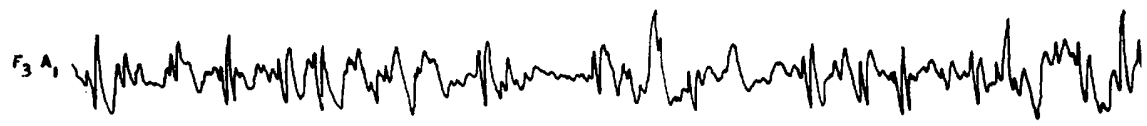

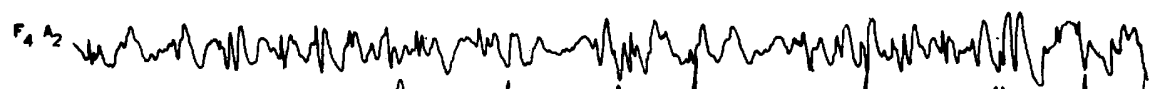

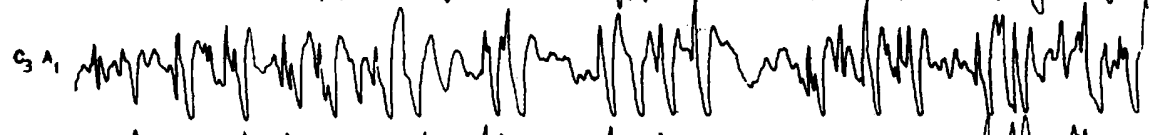

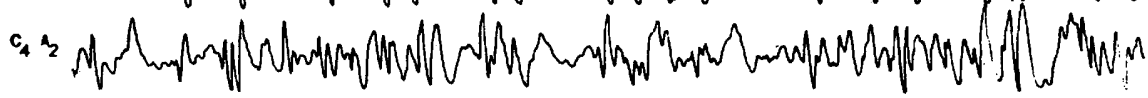

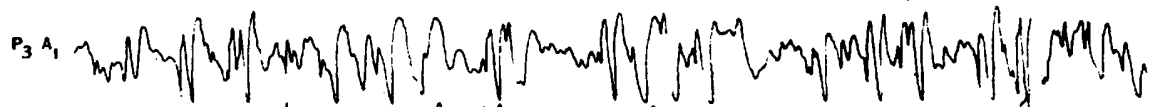

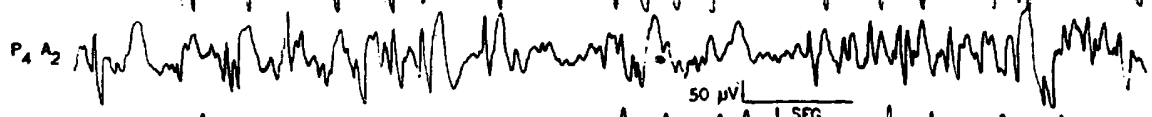

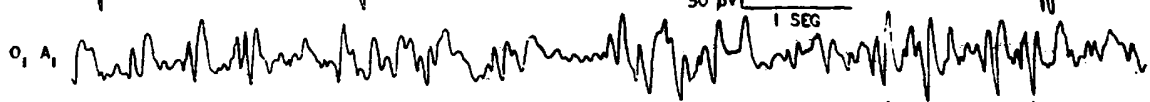

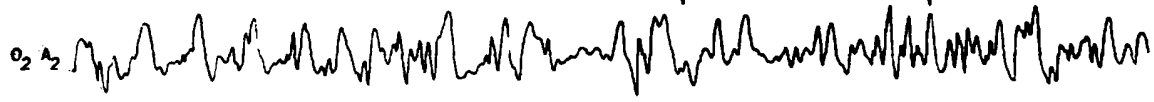

Fig. 2 - Caso S.R.M.O. - Sono barbitúrico. Após 10 meses de tratamento com difenilhidantoina. Atividade de fundo lenta $e$ irregular. Pontas e complexos ponta-ondas rápidas e lentas difusos, irregulares e pseudorrítmicos, com predomínio em áreas rolandico-parietais esquerdas.

$F_{3} A_{1}$

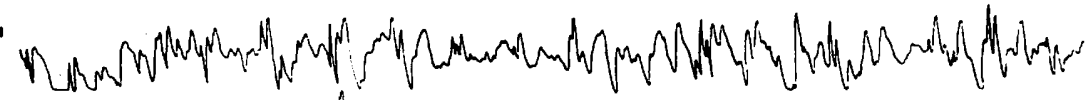

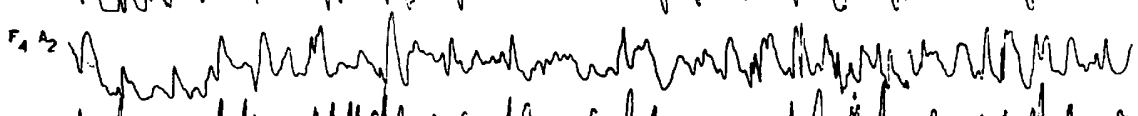

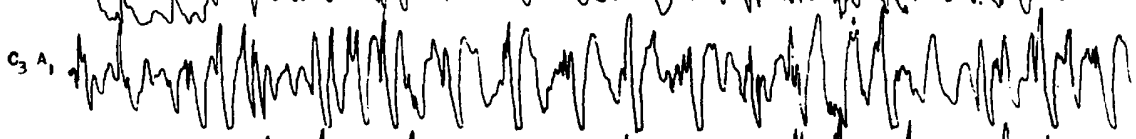

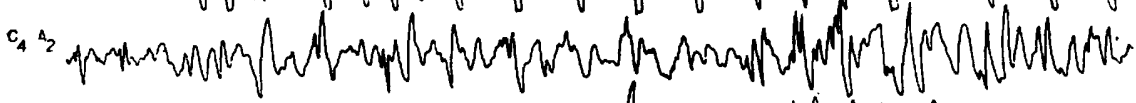

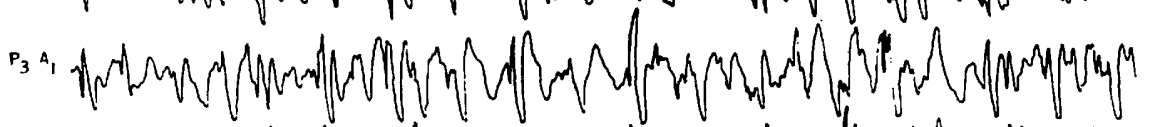

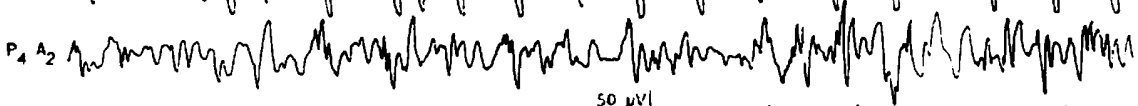

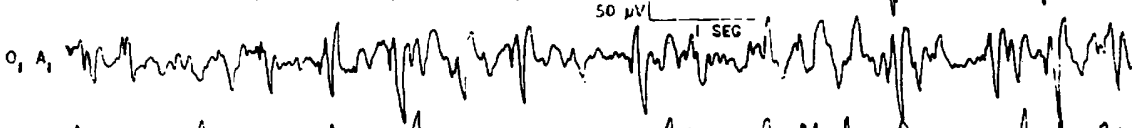

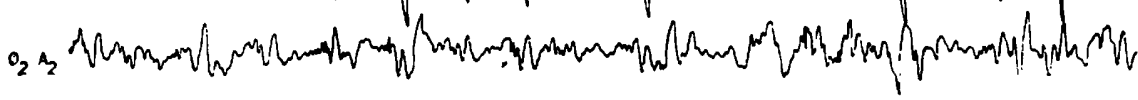

Fig. 3 - Caso S.R.M.O. - Sono barbitúrico. Exame realizado 14 dias após suspensão da difenilhidantoina. 


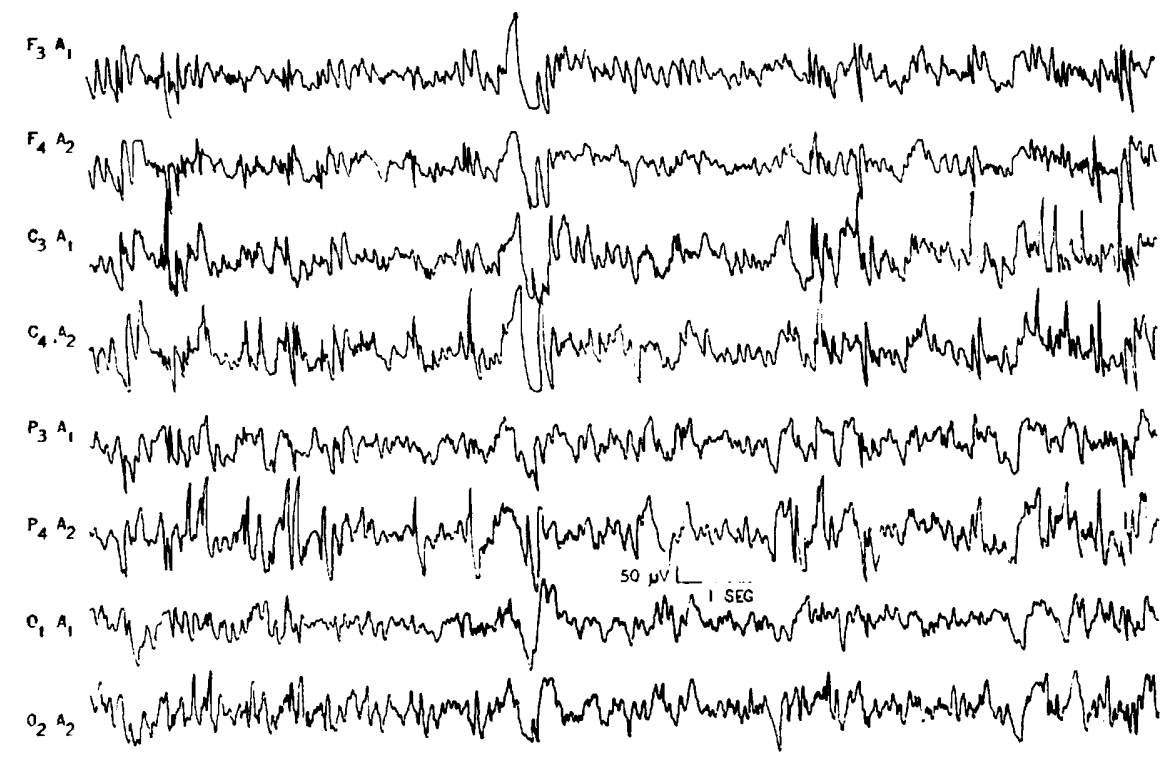

Fig. 4 - Caso S.R.M.O. - Sono barbitúrico. Exame realizado um mês após suspensĩo da difenilhidantoina.

com criação de novas zonas epileptógenas) ${ }^{1,5}, 7,11,13$. Tais fatos respondem pela eventual "malignização" de epilepsias consideradas, via de regra, benígnas: epilepsias hemigeneralizadas ${ }^{+,}{ }^{10}$, crises febrís ${ }^{8,20}$, epilepsias generalizadas com ponta-onda rápida ${ }^{14}$, epilepsias funcionais de maturação ${ }^{13}$.

O uso de medicações de ação predominante sobre uma das duas formas fundamentais de epilepsia, a convulsiva ou a não convulsiva, tem provocado o aparecimento de manifestações epilépticas de sentido oposto ao de seu campo habitual de ação $9,16,18,19$. Foi relatada a incidência de crises atônicas, graves pelas suas conseqüências, possivelmente causadas pelas terapêuticas utilizadas em pacientes com epilepsia generalizada comum ou com sindrome de Lennox ${ }^{15}$. A observação pessoal de um caso com epilepsia convulsiva generalizada e atividades paroxísticas focais múltiplas que, após administração de difenilhidantoina, passou a apresentar quadro de epilepsia hemigeneralizada não convulsiva com caracteristicas eletrencefalográficas de síndrome de Lennox evidencia agravamento iatrogênico. $\mathrm{O}$ novo quadro eletro-clínico surgiu meses após o início da terapêutica com difenilhidantoina quando a criança apresentava sinais de intolerância à droga. A retirada da medicação foi seguida pelo desaparecimento das manifestações clínicas adquiridas persistindo, embora atenuadas, as eletrencefalográficas. Depreende-se desta observação a necessidade da avaliação cuidadosa de pacientes nos quais se desenvolveram novas formas de epilepsias a fim de verificar se a reversibilidade do quadro é a regra. Nessa perspectiva é da maior 
importância investigar o aparecimento de encefalopatias epilépticas graves em crianças tratadas com medicações antiepilépticas, e se tal fato ocorreu, quais os tipos iniciais de epilepsia, estadios de maturação cerebral e terapêutica.

O distúrbio eletrencefalográfico inicial observado em nosso paciente era constituído por atividades paroxísticas focais múltiplas. Tais paroxismos, em particular os rolândicos, sāo encontrados em formas benignas de epilepsia ${ }^{2}{ }^{3}$. Fatos clínicos e eletrencefalográficos sugerem sua relação com as epilepsias generalizadas da infância. As pontas responderiam em nível cortical a um processo subcortical influenciado por fatores genéticos e relacionado com a maturação cerebral ${ }^{2}$. A exemplo de certas formas evolutivas malígnas das epilepsias generalizadas não convulsivas e hemigeneralizadas da infância 6, 1+. 10 não encontramos motivos para excluir a possibilidade da epilepsia funcional de maturação ${ }^{17}$ com focos múltiplos apresentar várias modalidades evolutivas na dependência da intensidade e da qualidade das agressões cerebrais pregressas, da incidência de novas agressões em determinados estados e maturação e, provavelmente, de condições inerentes ao indivíduo.

A observação pessoal de dois casos com descargas paroxísticas focais "de maturação" e com crises epilépticas, qualitativa e quantitativamente semelhantes às encontradas na sindrome de Lennox, controladas exclusivamente com derivados benzodiazepínicos demonstra a existência de formas de transição ${ }^{13}$.

Em pelo menos um caso há suspeita de indução de epilepsia grave e evolutiva por tratamento prolongado com drogas de ação anticonvulsivante em lactente ${ }^{12}$. Após 16 meses de tratamento de uma epilepsia generalizada convulsiva a criança apreesntou manifestações não convulsivas e ponta-ondas lentas difusas. Apesar de controladas as crises com drogas de ação predominante sobre as manifestações não convulsivas, recidiva posterior à publicação confirmou a instalação definitiva de sindrome de Lennox.

A ocorrência de epilpsia com particularidades malígnas na vigência de tratamento anticonvulsivo, causada provavelmente pelo desiquilibrio entre os mecanismos cerebrais responsáveis pelas manifestações convulsivas e não convulsivas, pode iniciar verdadeiro ciclo vicioso, com o surgimento de uma epilepsia polimorfa e rebelde. Impõe-se, portanto, a adoção de medidas amplas de proteção antiepiléptica em pacientes que demonstram labilidade excessiva na genese de suas crises.

\section{RESUMO E CONCLUSOES}

É relatada a incidência de epilepsia não convulsiva com características eletrencefalográficas de síndrome de Lennox em paciente de 4 anos de idade tratada com difenilhidantoina. A criança, ao iniciar o tratamento, era acometida por epilepsia convulsiva com atividades paroxísticas focais múltiplas no eletrencefalograma. 
Com base na observação de formas de transição entre epilepsias benignas c graves da infância destacou-se o papel da terapêutica no estabelecimento de epilepsia iatrogênica com características malígnas.

\section{S U M M A R Y}

\section{Undesirable effect of antiepileptic therapy in a patient with functional} maturational epilepsy

The incidence of non-convulsive epilepsy with electroencephalographic features of Lennox syndrome is related in a 4 years old patient treated with diphenyl-hydantoin. The child had convulsive epilepsy associated with focal multiple spikes in the electroencephalogram at the beginning of the treatment.

Based on the observation of transitional forms of benign and severe childhood epilepsies the role of the drug in the establishment of severe yatrogenic epilepsy is discussed.

\section{REFERENCIAS}

1. AICARDI, J. \& CHEvRIE, J. J. - Convulsive status epilepticus in infants and children. A study of 239 cases. Epilepsia (Amsterdam) 11:187, 1970.

2. BEAUSSART, M. - Benign epilepsy of children with rolandic (centro-temporal) paroxysmal foci. A clinical entity. Study of 221 (ases. Epilepsia (Amsterdam) 13:795, 1972.

3. BLOM, S.; HEJBEL, J. \& BERGFORS, P. G. - Benign epilepsy of children with centro-temporal EEG foci. Prevalence and follow-up study of 40 patients. Epilpsia (Amsterdam) 13:609, 1972.

4. GASTAUT, H.; VIGOUROUX, M.; TREVISAN, C. \& REGIS, H. - Le syndrome "hémiconvulsion-hémiplégie-épilepsie" (Syndrome HHE de l'enfant). Rev. Neurol. (Paris) 97:37, 1957.

5. GASTAUT, H. \& TASSINARI, C. A. - Triggering mechanisms in epilepsy. The electroclinical point of view. Epilepsia (Amsterdam) 7:85, 1966.

6. GASTAUT, H.; ROGER, J.; SOULAYROL, R.; TASSINARI, C. A.; RÉGIS, H.; DRAVET, C.; BERNARD, N, \& SAINT-JEAN, M. - Childhood epileptic encephalopathy with diffuse slow spike-waves (otherwise known as "Petit mal variant" or Lennox syndrome. Epilepsia (Amsterdam) 7:139, 1966.

7. GASTAUT, H.; ROGER, J. \& LOB, H. - Les Etats de Mal Épileptiques. Masson et cir, Paris, 1967.

\&. LINDSAY, J. M. M. - Genetics and epilepsy: a model from critical path analysis. Epilepsia (Amsterdam) 12:47, 1971.

9. LISON, M. P. - Contribuição ao estudo da ação de derivados benzodiazepinicos na epilepsia. Tese, Ribeirão Preto, 1969.

10. LISON, M. P. - Bissincronia secundária na síndrome de Lennox. Arq. NeuroPsiquiat. (São Paulo) 28:235, 1970.

11. LISON, M. P. - Espasmos em flexão: estudo clínico longitudinal de pacientes tratados com nitrazepam (Mogadon). Arq. Neuro-Psiquiat. (São Paulo) 28:336, 1970.

12. LISON, M. P.; MOURA RIBEIRO, M. V. \& MEGA, D. - Complicacão rara e reversivel de sindrome convulsiva: hipotonia generalizada e alteracōes da atividade intelectual. Arq. Neuro-Psiquiat. (São Paulo) 25:30. 1967. 
13. LISON, M. P. \& SPECIALI, J. G. - Sindrome epiléptica grave na infância com descargas paroxísticas focais "de maturação". Estudo clínico-eletrencefalográfico longitudinal. Arq. Neuro-Psiquiat. (São Paulo) 29:164, 1971.

14. OLLER-DAURELLA, L.; DINI, J. \& MARQUEZ, J. - Las encefalopatias epileptógenas infantiles difusas no especificas, compreendido el sindrome de Lennox. Bol. Soc. Cat. Ped. 29:3, 1968.

15. OlLER-DAURELlA, L. - Commentarios a ciertos aspectos clínicos-EEG poco frecuentes en las crises atonicas: los "status" atonicos y las ausências con pérdida del tono postural unilateral. Rev. Esp. Oto. Neurol. Oft. Neurocir. 27:303, 1968.

16. PRIOR, P. F.; MCLAINE, C. N.; SCOTT, D. F. \& LAURENCE, B. M. - Tonic status epilepticus precipitated by intravenous diazepam in a child with Petit Mal Status. Epilepsia (Amsterdam) 13:467, 1972.

17. SOREL, L. \& RUCQUOY-PONSAR, M. - L'épilepsie fonctionelle de maturation. Apport des montages verticaux dans le diagnostic de cette forme d'épilepsie. Rev. Neurol, (Paris) 121:288, 1969.

18. SUBIRANA, A. \& OLLER-DAURELLA, L. - L'aggravation de certains cas d'épilepsie soit par les traitements antiépileptiques, soit par les thérapeutiques couramment associées. Rev. Neurol. (Parßs) 110:293, 1963.

19. TASSINARI, C. A.; DRAVET, C.; ROGER, J.; CANO, J. P. \& GASTAUT, H. Tonic status epilepticus precipitated by intravenous benzodiazepine in five patients with Lennox-Gastaut syndrome. Epilepsia (Amsterdam) 13:421, 1972.

20. TAYLOR, D. C. \& OUNSTED, C. - Biological mechanisms influencing the outcome of seizures in response to fever. Epilepsia 12:33, 1971.

Departamento de Neuropsiquiatria e Psicologia Médica - Faculdade de Medicina - 14100 Ribeirão Preto, SP Brasil. 